\title{
Application de la méthode des points quadrats au suivi de la dynamique des pâturages soudano-sahéliens du Cameroun
}

\author{
Joseph ONANA ${ }^{1 *}$, Jean Pierre MVONDO AWONO ${ }^{2}$, Dalil ABBA ${ }^{3}$ et Jean François \\ Bruno OTTOU ${ }^{1}$ \\ ${ }^{I}$ Institut de Recherches Agricoles pour le Développement (IRAD), Direction de la valorisation, B.P. 2123 \\ Yaoundé, Cameroun. \\ ${ }^{2}$ CEDEC / Université de Dschang, Faculté d'Agronomie et des Sciences Agricoles (FASA), B.P. 222 Dschang, \\ Cameroun. \\ ${ }^{3}$ Ministère de la Recherche Scientifique et de l'Innovation, B.P. 1457 Yaoundé, Cameroun. \\ *Auteur correspondant, Tél. cell. 99795639 ; E-mail: onanajo2003@yahoo.fr
}

\section{RESUME}

L'étude de la structure du couvert herbacé de quelques formations pastorales des secteurs phytogéographiques soudano-sahéliens et sahélo-soudaniens du Cameroun a été faite par la méthode des points quadrats alignés dans le but de déterminer le nombre de lignes permanentes statistiquement nécessaires pour le suivi de la dynamique de cette végétation. Les résultats obtenus montrent qu'au seuil de probabilité de $5 \%$, une seule ligne permanente est nécessaire pour suivre la dynamique des groupements à Aristida kerstingii, Heteropogon contortus, Andropogon pinguipes, et que deux lignes sont requises pour les groupements à Andropogon gayanus, Loudetia simplex, et Loudetia togoensis. Trois lignes sont nécessaires pour les groupements à Hyparrhenia rufa et Echinochloa pyramidalis. Pour avoir une marge de sécurité et tenir compte de l'évolution possible des divers groupements, nous proposons d'effectuer pour le suivi de l'ensemble des parcours étudiés des observations sur cinq lignes permanentes.

(C) 2008 International Formulae Group. All rights reserved.

Mots clés: Cameroun, Formation pastorale, points contacts, dynamique de la végétation.

\section{INTRODUCTION}

Le Cameroun, de concert avec la Communauté internationale, s'est engagé depuis le premier sommet de la terre (RIO, 1992) à renforcer le suivi de la coordination efficace des interventions en matière de la protection et la gestion rationnelle de l'environnement et des ressources naturelles pour un développement durable. Les indications actuelles sur l'état et les tendances de l'environnement, et les menaces qui l'affectent, sont pour la plupart d'ordre qualitatif et les données quantitatives standardisées font défaut dans la plus part des pays en voie de développement. Conscient de ce manquement, le Réseau Africain d'Information Environnementale (RAIE) est né afin de contribuer à la mise en oeuvre des objectifs des composantes environnementales du Nouveau Partenariat pour le Développement de l'Afrique (NEPAD).

En en ce qui concerne le suivi du couvert végétal au Cameroun, aucun système de suivi n'a été mis en œuvre malgré des travaux réalisés en 1975 et entre 1980 et 1990 , visant à établir une méthodologie permettant de cartographier périodiquement la couverture végétale à partir de l'interprétation d'images satellite, afin d'en suivre l'évolution au cours du temps. L'absence de répétitions systématiques de ces opérations selon des modalités préétablies ne permet pas de parler de système de suivi.

Dans le cas du monitoring des pâturages, en dehors de l'aspect spatialisation qui peut être suivi à partir des images satellitaires, il importe aussi de suivre l'évolution de la qualité du couvert herbacé 
qui est tributaire de la capacité de charge et qui se définit comme étant la quantité d'animaux de $250 \mathrm{~kg}$ (=1 UBT) que peut supporter le pâturage sans se dégrader.

Des travaux phytosociologiques ont été réalisés dans la zone soudano-sahélienne du Cameroun au cours des années 1990 à 1995 (Donfack, 1993; Onana 2005) en vue de caractériser les groupements végétaux tant ligneux qu'herbacés des terres de parcours (Tableau 1). Huit principaux groupements d'intérêt pastoral ont ainsi été mis en évidence. Dans le cadre du monitoring environnemental (ME) et de l'information environnemental (IE), les méthodes de suivi de la dynamique des pâturages, longtemps mise au point par les gestionnaires des écosystèmes pâturés, ont souvent été mise en œuvre de façon partielle en zone soudanosahélienne du Cameroun. La présente étude qui utilise la méthode des points quadrats vise à mettre à la disposition des utilisateurs et des gestionnaires des pâturages naturels un référentiel technique de suivis écologiques susceptibles de permettre à tous les acteurs de générer des données fiables et échangeables pour le monitoring de la ressource pastorale.

\section{MATERIEL ET METHODES Matériel}

La zone d'étude est comprise entre $8^{\circ} 30^{\prime}$ et $12^{\circ}$ latitude Nord. Elle appartient au domaine phytogéographique soudanien avec une pluviosité moyenne de $1000 \mathrm{~mm} \cdot \mathrm{an}^{-1}$ et une humidité relative de $80 \%$ en saison des pluies et $40 \%$ en saison sèche. Le substratum géologique est formé de roches diverses : grès, granite, gneiss, micaschistes, gabbro. Sept classes de sol sont rencontrées (Sols minéraux bruts, peu évolués, vertisols, brunifiés, ferrugineux, hydromorphes, et sodiques).

\section{Méthodes}

L'étude de la structure horizontale de huit principaux groupements herbacés des terres de parcours (Tableau 1) décrits dans la région soudano-sahélienne du Cameroun (Onana, 1995) a été faite par la méthode des points quadrats de Levy et Madden (1933), méthode qui a été reprise par de nombreux auteurs (Boudet, 1984 ; Daget, 1971 ; Daget et Poissonnet, 1971, 1991; Daget et al., 1995 ; Gaston, 1992 ; PNUD, 2006).

Cette méthode consiste à tendre un double décamètre au-dessus du toit du tapis herbacé et tous les $20 \mathrm{~cm}$, une tige fine en fer à béton est enfoncée verticalement dans la végétation. Toutes les espèces en contact avec la tige sont enregistrées sur des bordereaux préétablis. Par convention, chaque espèce est notée une seule fois à chacun des 100 points de lecture afin de donner une meilleure image de la proportion des espèces en projection au sol.

Cinq lignes au minimum ont été distribuées au hasard dans chaque groupement afin d'estimer avec suffisamment de précision la composition des pâturages.

Les relevés ont été réalisés au mois d'août 2002 pendant que la majeure partie des espèces était déjà en fleurs, donc facilement identifiable.

\section{Analyse des données}

L'estimation de la fréquence absolue encore appelée fréquence spécifique (Fs) de chaque espèce sur chaque relevé a été obtenue par comptage du nombre de fois que chaque espèce apparaît sur les 100 points de lecture afin d'identifier les espèces dominantes.

La proportion de chaque espèce dans le relevé a été exprimée en pourcentage par rapport au total des proportions de toutes les espèces par la formule :

$$
C S i=\frac{F S i}{\sum_{i=1}^{n} F S i}
$$

Cette valeur relative de chaque espèce est appelée contribution spécifique de l'espèce $\mathrm{i}$ (CSi).

Le nombre de lignes de points quadrats à mettre en place dans chaque groupement pour valider le sondage est statistiquement déterminé par l'intervalle de confiance (IC) calculé à partir de l'effectif cumulé ligne par ligne, des contacts de l'espèce dominante sur l'effectif cumulé de tous les contacts enregistrés pour l'ensemble des espèces (Boudet, 1984). L'effet du hasard est considéré comme éliminé lorsque la précision atteinte est voisine de $5 \%$.

L'intervalle de confiance est calculé par la formule (Boudet, 1984) :

$P= \pm 2 \times \sqrt{\frac{n(N-n)}{N^{3}}}$ 
où : $\mathrm{N}$ est l'effectif cumulé ligne par ligne des contacts de l'ensemble des espèces, et n l'effectif cumulé ligne par ligne des contacts de l'espèce dominante.

La fréquence relative exprimée en pourcentage peut alors s'écrire selon la formule suivante:

$\mathrm{CSi}(\%) \pm \mathrm{P}(\%)$.

\section{RESULTATS}

Bien que l'échantillonnage ne soit pas identique, (5 à 12 lignes selon les groupements), la précision des mesures pour chaque relevé (ligne d'observation) varie :

- pour le groupement a Aristida kerstingii de 6,02 à $7,68 \%$,

- pour le groupement à Andropogon pinguipes de 4,64 à 7,64 \%,

- pour le groupement à Loudetia simplex ces variations vont de 5,9 à 10,64\% pour le sous-groupement à Loudetia simplex sur sables humides des bas-fonds, de 5,45 à $6,17 \%$ pour celui des sols minéraux bruts et de 4,07 à 4,59 pour celui des basfonds faiblement inondés,

- pour le groupement à Brachiaria jubata de 4,58 à $5,72 \%$,

- pour le groupement à Setaria pumila de 4,9 à $5,36 \%$.

On note, d'après les résultats qui précèdent, une hétérogénéité marquée des groupements à Andropogon pinguipes et du sous-groupement à Loudetia simplex sur sable humide des basfonds.
Le nombre de relevés à prendre en compte pour atteindre une précision du sondage de $5 \%$ (Tableau 2) est :

- d'une ligne pour les groupements à Andropogon pinguipes, à Setaria pumila, et à Loudetia simplex des zones humides, - de deux lignes pour les groupements à Hyparrhenia rufa, à Loudetia simplex des stations mésophiles, à Brachiaria jubata, et à Loudetia togoensis,

- de trois lignes pour les groupements à Aristida kerstingii, et à Echiochloa pyramidalis,

- et de quatre lignes pour le sousgroupement à Loudetia simplex sur sol minéraux bruts.

\section{DISCUSSION}

Certains des groupements étudiés en zone soudano-sahélienne du Cameroun ont également été décrits au Tchad (Gaston et al., 1977). Une étude comparable dans ce pays voisin serait de nature à permettre une généralisation des méthodes de suivi écologique des pâturages de la zone aride et semi-aride d'Afrique Centrale. Il est cependant important de noter que dans la zone guinéenne plus au sud (plateau de l'Adamaoua), la méthode des points quadrats a subi des adaptations pour pouvoir être appliquée (Rippstein, 1985). En effet, dans ces zones, les Andropogonées atteignent des tailles impressionnantes (plus de 2 mètres à

Tableau 1: Spectre des groupements pastoraux étudiés selon le type de sol en zone soudanosahélienne et production potentielle de phytomasse aérienne.

\begin{tabular}{|c|c|c|c|c|c|c|c|c|}
\hline \multirow[t]{2}{*}{ Groupement } & \multicolumn{7}{|c|}{ Classe de sol } & \multirow[t]{2}{*}{ P (t.M.S/ha) } \\
\hline & I & II & III & VII & IX & $\mathbf{X I}$ & XII & \\
\hline Andropogon pinguipes & +++ & + & ++ & - & +++ & - & - & $7,90 \pm 1,20$ \\
\hline Aristida kerstingii & + & - & + & + & ++ & - & ++ & $3,70 \pm 0,80$ \\
\hline Brachiaria jubata & - & - & + & ++ & - & +++ & - & $4,80 \pm 0,60$ \\
\hline Echinochloa pyramidalis & - & - & - & - & - & +++ & - & $8,08 \pm 1,07$ \\
\hline Hyparrhenia rufa & - & - & - & - & - & ++ & - & $7,80 \pm 0,90$ \\
\hline Loudetia simplex & +++ & - & - & - & - & +++ & - & $5,80 \pm 2,20$ \\
\hline Loudetia togoensis & + & - & + & - & +++ & - & + & $3,80 \pm 0,70$ \\
\hline Schizachyrium exile & - & + & - & - & + & +++ & + & $3,20 \pm 0,66$ \\
\hline Setaria pumila & + & + & +++ & + & +++ & - & + & $3,10 \pm 0,90$ \\
\hline
\end{tabular}


Tableau 2: Evaluation du nombre de lignes nécessaires pour l'étude par points quadrats des groupements herbacés représentatifs dans les terres de parcours.

\begin{tabular}{|c|c|c|c|c|c|c|}
\hline Groupement & $\begin{array}{c}\begin{array}{c}\text { Numéros de } \\
\text { lignes }\end{array} \\
\end{array}$ & $\mathbf{n}^{\prime}$ & $\mathbf{N}^{\prime}$ & $\mathbf{F r} \mathbf{r}^{\prime}$ & $\mathbf{P}(\%)$ & $\mathbf{P}^{\prime}(\%)$ \\
\hline \multirow{5}{*}{ Andropogon pinguipes } & 1 & 99 & 366 & 27,05 & 4,64 & 4,64 \\
\hline & 2 & 189 & 568 & 33,27 & 6,99 & 3,95 \\
\hline & 3 & 280 & 767 & 36,51 & 7,06 & 3,48 \\
\hline & 4 & 375 & 939 & 39,94 & 7,58 & 3,20 \\
\hline & 5 & 461 & 1110 & 41,53 & 7,65 & 2,96 \\
\hline \multirow{12}{*}{ Aristida kerstingii } & 1 & 100 & 193 & 51,81 & 7,19 & 7,19 \\
\hline & 2 & 199 & 399 & 49,87 & 6,96 & 5,01 \\
\hline & 3 & 281 & 620 & 45,32 & 6,50 & 4,00 \\
\hline & 4 & 367 & 869 & 42,23 & 6,03 & 3,35 \\
\hline & 5 & 467 & 1015 & 46,01 & 7,69 & 3,13 \\
\hline & 6 & 565 & 1190 & 47,48 & 7,50 & 2,90 \\
\hline & 7 & 665 & 1369 & 48,58 & 7,42 & 2,70 \\
\hline & 8 & 765 & 1542 & 49,61 & 7,51 & 2,55 \\
\hline & 9 & 839 & 1754 & 47,83 & 6,55 & 2,39 \\
\hline & 10 & 939 & 1890 & 49,68 & 7,57 & 2,30 \\
\hline & 11 & 1038 & 2051 & 50,61 & 7,67 & 2,21 \\
\hline & 12 & 1128 & 2257 & 49,98 & 6,91 & 2,10 \\
\hline \multirow{5}{*}{ Brachiaria jubata } & 1 & 83 & 276 & 30,07 & 5,52 & 5,52 \\
\hline & 2 & 179 & 572 & 31,29 & 5,44 & 3,88 \\
\hline & 3 & 249 & 828 & 30,07 & 5,57 & 3,19 \\
\hline & 4 & 333 & 996 & 33,43 & 7,72 & 2,99 \\
\hline & 5 & 421 & 1138 & 36,99 & 8,15 & 2,86 \\
\hline \multirow{5}{*}{ Echinochloa pyramidalis } & 1 & 100 & 149 & 67,11 & 7,70 & 7,70 \\
\hline & 2 & 192 & 337 & 56,97 & 7,29 & 5,39 \\
\hline & 3 & 282 & 517 & 54,55 & 7,45 & 4,38 \\
\hline & 4 & 371 & 689 & 53,85 & 7,62 & 3,80 \\
\hline & 5 & 460 & 928 & 49,56 & 6,25 & 3,28 \\
\hline \multirow{5}{*}{ Hyparrhenia rufa } & 1 & 98 & 193 & 50,78 & 7,20 & 7,20 \\
\hline & 2 & 196 & 402 & 48,76 & 6,90 & 4,99 \\
\hline & 3 & 296 & 631 & 46,91 & 6,55 & 3,97 \\
\hline & 4 & 388 & 835 & 46,47 & 6,97 & 3,45 \\
\hline & 5 & 480 & 1065 & 45,07 & 6,46 & 3,05 \\
\hline \multirow{5}{*}{$\begin{array}{l}\text { Loudetia simplex (faciès à } \\
\text { Hyperthelia dissoluta) }\end{array}$} & 1 & 59 & 211 & 27,96 & 6,18 & 6,18 \\
\hline & 2 & 119 & 458 & 25,98 & 5,46 & 4,10 \\
\hline & 3 & 174 & 698 & 24,93 & 5,43 & 3,27 \\
\hline & 4 & 242 & 912 & 26,54 & 6,37 & 2,92 \\
\hline & 5 & 305 & 1118 & 27,28 & 6,42 & 2,66 \\
\hline \multirow{7}{*}{$\begin{array}{l}\text { Loudetia simplex (faciès à } \\
\text { Schizachyrium sanguineum) }\end{array}$} & 1 & 44 & 104 & 42,31 & 9,69 & 9,69 \\
\hline & 2 & 90 & 203 & 44,33 & 10,03 & 6,97 \\
\hline & 3 & 136 & 291 & 46,74 & 10,65 & 5,85 \\
\hline & 4 & 164 & 409 & 40,10 & 7,83 & 4,85 \\
\hline & 5 & 225 & 635 & 35,43 & 5,91 & 3,80 \\
\hline & 6 & 286 & 861 & 33,22 & 5,91 & 3,21 \\
\hline & & 227 & & & & \\
\hline
\end{tabular}




\begin{tabular}{lcccccc} 
& 7 & 351 & 1099 & 31,94 & 5,78 & 2,81 \\
& 8 & 375 & 1210 & 30,99 & 7,81 & 2,66 \\
\hline \multirow{2}{*}{ Loudetia simplex (Faciès à } & $\mathbf{1}$ & $\mathbf{6 8}$ & $\mathbf{3 1 8}$ & $\mathbf{2 1 , 3 8}$ & 4,60 & $\mathbf{4 , 6 0}$ \\
Spermacoce filifolia) & 2 & 137 & 685 & 20,00 & 4,08 & 3,06 \\
& 3 & 198 & 1045 & 18,95 & 3,95 & 2,42 \\
& 4 & 268 & 1317 & 20,35 & 5,30 & 2,22 \\
& 5 & 339 & 1469 & 23,08 & 8,09 & 2,20 \\
\hline Loudetia togoensis & 1 & 88 & 284 & 30,99 & 5,49 & 5,49 \\
& $\mathbf{2}$ & $\mathbf{1 3 6}$ & $\mathbf{4 9 7}$ & $\mathbf{2 7 , 3 6}$ & 5,73 & $\mathbf{4 , 0 0}$ \\
& 3 & 188 & 781 & 24,07 & 4,59 & 3,06 \\
& 4 & 278 & 973 & 28,57 & 7,20 & 2,90 \\
Schyzachyrium exile & 5 & 368 & 1209 & 30,44 & 6,32 & 2,65 \\
\hline & 1 & 80 & 271 & 29,52 & 6,42 & 5,54 \\
& $\mathbf{2}$ & $\mathbf{1 8 0}$ & $\mathbf{4 5 2}$ & $\mathbf{3 9 , 8 2}$ & 5,54 & $\mathbf{4 , 6 1}$ \\
& 3 & 269 & 797 & 33,75 & 7,39 & 3,35 \\
& 4 & 358 & 1004 & 35,66 & 4,71 & 3,02 \\
& 5 & 452 & 1286 & 35,15 & 6,88 & 2,66 \\
\hline
\end{tabular}

$\mathrm{n}^{\prime}$ = fréquence cumulée ligne par ligne de l'espèce dominante, $\mathrm{N}^{\prime}=$ fréquence cumulée ligne par ligne de l'ensemble des espèces, $\mathrm{Fr}^{\prime}=$ Fréquence cumulée ligne par ligne de l'espèce dominante en $\%, \mathrm{P}(\%)=$ Précision atteinte sur chaque relevé, $\mathrm{P}^{\prime}(\%)=$ Précision cumulée ligne par ligne. En gras = référence au nombre de ligne pour atteindre une précision inférieure ou égale à $5 \%$.

épiaison), ce qui ne permet plus de tendre le mètre au-dessus du toit de la végétation. Il est dont nécessaire soit de reconnaître toutes les plantes au stade juvénile pour réaliser les relevés avant le stade d'épiaison, soit de rabattre la végétation juste avant l'analyse.

Compte tenu du nombre variable de relevés nécessaire d'un groupement à un autre pour atteindre la précision de 5\% (1 à 4), il paraît nécessaire de recommander la mise en place de 5 relevés (lignes) dans le cadre d'un monitoring des ressources pastorales de la zone soudano-sahélienne du Cameroun. Un tel échantillon a pour avantage de couvrir la multitude de groupements végétaux d'intérêt pastoral rencontré dans la région et permettrait d'harmoniser la méthodologie de collecte de données sur l'évolution des pâturages. Les résultats obtenus ici sont conformes à ceux obtenus dans certaines formations pastorales à écologie comparable de Côte-d'Ivoire (Gaston et al., 1977 ; Gaston, 1992).

Si la méthode quantitative par points quadrats est parmi celles les plus utilisées actuellement, elle n'est pas la seule à pouvoir donner satisfaction. Il existe en effet des indicateurs biologiques pouvant permettre de donner des informations sur l'état d'évolution d'un pâturage. Ces bio-indicateurs sont actuellement en cours d'élaboration et d'harmonisation pour la zone soudanosahélienne d'Afrique Centrale (Onana et al, com. pers.). On peut par exemple citer Hyptis suaveolens, Hyptis spicigera, Sida rhombifolia et Brachiaria xantholeuca qui sont des espèces indicatrices du surpâturage. La mise en place d'une composante pastorale dans les bases de données environnementales est recommandée et pourrait utiliser l'outil élaboré au cours des présents travaux.

\section{Conclusion}

Les relevés par points quadrats effectués dans les pâturages soudanosahéliens du Cameroun constituent le préalable pour la mise en place d'un système de monitoring de ces écosystèmes actuellement surexploités en raison de la 
diminution progressive des terres de parcours en relation avec l'augmentation constante des nouvelles terres agricoles d'une part et le surpâturage d'autre part. La présente étude montre qu'il est nécessaire de mettre un maximum de cinq relevés par points quadrats dans n'importe quel pâturage de la région afin d'en suivre l'évolution ; un surplus de relevés n'étant plus statistiquement accompagné d'un gain significatif de précision sur les données collectées. Les résultats obtenus lors de cette étude se doivent d'être valorisés dans le monitoring environnemental actuellement en cours de montage au sein du Ministère de l'Environnement et de la Protection de la Nature au Cameroun pour prendre en compte les écosystèmes pâturés.

\section{REFERENCES BIBLIOGRAPHIQUES}

Boudet G. 1984. Manuel sur les Pâturages Tropicaux et les Cultures Fourragères (4e édn, révisée). IEMVT: MaisonsAlfort ; $266 \mathrm{p}$.

César J. 1971. Etude quantitative de la strate herbacée de la savane de Lamto (Moyenne Côte-d'Ivoire). Thèse doctorat 3 e cycle, Paris VI, 95 p.

Daget P, Bille JC, Corn, Gaston A, Konaté T. 1995. Organisation du suivi de la production végétale dans les pâturages : Exemples du Mali et de l'Ethiopie. Parcours Demain. Numéro spécial. Actes $\mathrm{du}$ séminaire international du réseau parcours, Tabarka (Tunisie), 13-15 octobre 1994 : 67-71.

Daget P, Poissonnet J. 1971. Une méthode d'analyse phytosociologique des prairies. Ann. Agron., INRA, Paris. 22(1) : 5 - 41.

Daget P, Poissonnet J. 1991. Prairies Permanentes et Pâturages. Méthodes d'Etude. Inst. Bot.: Montpellier; 331p.

Gaston A, Dulieu D, Lamarque G. 1977. Synthèse Agropastorale du Bassin du Lac Tchad. IEMVT, Maisons-Alfort/ NDJAMENA, CBLT, N'djamena ; 263 p.

Donfack P. 1993. Etude de la dynamique de la végétation après abandon de la culture au
Nord-Cameroun. Thèse Doct. 3e cycle, Université de Yaoundé, 185p + annexes.

Gaston J. 1992. La production Biologique des Savanes de Côte-d'Ivoire et son Utilisation par l'Homme. Biomasse, Valeur Pastorale et Production Fourragère. CIRAD-IEMVT : MaisonsAlfort ; $671 \mathrm{p}$.

Levy B, Madden E. 1933. The point method of pasture analysis. New Leaf. Jr. Arg., 46: $267-279$.

Onana J. 1995. Les savanes soudanosahéliennes du Cameroun: analyse phytoécologique et utilisation pastorale. Thèse Doct. Université de Rennes 1, 155 p.

Onana J, Béchir A, Kilembé D. 2007. Bioindicateurs de dégradation des parcours en zone semi-aride d'Afrique Centrale : perception paysanne et rigueur scientifique (com. pers.), $12 \mathrm{p}$.

PNUD (Programme des Nations Unies pour le Développement). 2006. L'avenir de l'Environnement en Afrique: notre Environnement, notre Richesse. PNUD; $542 \mathrm{p}$.

Poissonnet J, César J. 1972. Etude Comparée de Diverses Méthodes d'Analyse de la Végétation. Doc. $\mathrm{N}^{\circ}$ 50. CNRS/CEPE: Montpellier; $120 \mathrm{p}$.

Poissonnet J, César J. 1972. Structure spécifique de la strate herbacée dans la savane à palmier rônier de Lamto, Côte d'Ivoire. Ann. Univ. Abidjan, F, 5: 577601.

Rippstein G. 1985. Etude sur la Végétation de l'Adamaoua: Evolution, Conservation, Régénération d'un Ecosystème Pâturé au Cameroun. IEMVT: Maisons-Alfort; $367 \mathrm{p}$.

Yonkeu S. 1991. Evolution d'un écosystème sous l'effet d'une forte charge animale dans la région de l'Adamaoua au Cameroun. IV ${ }^{\text {th }}$ International Rangeland Congress, Montpellier, France, pp.186188. 